\title{
The Role of Striate Cortex in Visual Function of the Cat
}

\author{
Tatiana Pasternak, ${ }^{1}$ Judith Tompkins, ${ }^{1}$ and Carl R. Olson ${ }^{2}$ \\ 1 Department of Neurobiology and Anatomy, and Center for Visual Science, University of Rochester, Rochester, \\ New York 14627 and ${ }^{2}$ Department of Anatomy, College of Dental Surgery, University of Maryland at Baltimore, \\ Baltimore, Maryland
}

\begin{abstract}
We examined the contribution of area 17 to visual function in two cats whose fixation was monitored by means of scleral search coils. Ibotenic acid lesions were made within the physiologically identified representation of the lower left visual field of area 17. In a detection task in which the cats simply indicated the presence or absence of a vertical grating, contrast sensitivity loss was greatest at middle spatial frequencies with no loss in spatial resolution. However, when cats were required to discriminate between vertical and horizontal gratings, sensitivity loss was profound at both middle and high spatial frequencies with an octave loss of spatial resolution. This greater loss was not due to disrupted orientation discrimination since sensitivity to the orientation of coarse gratings was unaffected in the lesioned hemifield. We also found deficits in the ability to discriminate the direction of grating motion, but only at higher spatial and lower temporal frequencies.
\end{abstract}

The role of area 17 in perceiving the global motion of complex patterns was also studied with high contrast, dynamic random dots drifting at high speeds. Paradoxically, area 17 lesion improved the perception of global motion. This improvement was eliminated by spatially filtering the dot patterns to remove high spatial frequencies, suggesting that the lesion has enhanced performance by interfering with masking by high spatial frequencies.

Our results demonstrate that the performance of traditional detection tasks may be insensitive to the effects of area 17 lesions. Discrimination tasks, on the other hand, revealed that area 17 neurons play a major role in the perception of higher spatial frequency stimuli as long as they move or flicker at low rates, but contribute little to these functions when the stimuli are coarse and move at high speeds.

[Key words: visual cortex, area 17, contrast sensitivity, orientation discrimination, direction discrimination, $d y-$ namic random dots, motion, cat]

\footnotetext{
Received May 24, 1994; revised Aug. 22, 1994; accepted Sept. 9, 1994

This work was supported by National Eye Institute Grants EY06175, EY05911, and EY01319 (Core Grant to the Center for Visual Science), and NINDS Grant NS27287. We thank John Maunsell for assistance and the use of the recording laboratory, and Larry Ota and Kathy Lazaroff for excellent technical assistance. We are also grateful to Emil Rainero for developing the computer software for stimulus generation and the control of experiments, Dorothy Flood for help with histological analysis, and William Merigan for comments on the manuscript.

Correspondence should be addressed to Tatiana Pasternak at the above address.

Copyright (C) 1995 Society for Neuroscience 0270-6474/95/151940-11\$05.00/0
}

In contrast to the $\mathrm{P}$ and $\mathrm{M}$ pathways of primates, which project to different layers within a single visual area, the $\mathrm{X}$ and $\mathrm{Y}$ pathways of the cat terminate largely in different cortical areas. $\mathrm{X}$-cells project almost exclusively to striate cortex, while area 18 receives its major input from Y-cells (Stone, 1983). The segregation is not perfect, however, insofar as some Y-cells probably project to area 17 (Stone and Dreher, 1973; Humphrey et al., 1985; Dreher et al., 1992; but see Ferster, 1988a).

Despite the fact that $X$ and $Y$ input pathways are not confined absolutely to areas 17 and 18, respectively, and despite the fact that areas 17 and 18 are strongly interconnected, it is clear that neurons in area 17 tend to have $\mathrm{X}$-like properties and those in area 18, Y-like properties. Thus, area 17 neurons prefer spatial frequencies that are a factor of three higher than those preferred by area 18 neurons (Movshon et al., 1978; Ferster and Jagadeesh, 1991; Burke et al., 1992). The two areas also differ in their temporal frequency response, with area 18 neurons responding to higher temporal frequencies than neurons in area 17. Thus, in cats, the first stages of cortical processing of $X$ and $\mathrm{Y}$ signals appear to be largely segregated in distinct areas. This pattern of cortical termination of the two pathways makes the cat a convenient animal model for lesion studies of the contribution of different ascending pathways to the processing of motion and form.

Despite a wealth of information about the physiological properties of area 17 and 18 neurons, little is known of their respective roles in visual function. A number of studies have used lesions to cxaminc the joint contribution of areas 17 and 18 to form perception, spatial resolution, and spatial contrast sensitivity (Sprague et al., 1977; Berkley and Sprague, 1979; Lehmkuhle et al., 1982). However, they could not separate the contributions of these areas to visual function because areas 17 and 18 are contiguous and share a representation of the vertical meridian (Tusa et al., 1979). In this study we examined the separate contributions of these areas to visual function by limiting lesions to portions of area 17 away from the vertical meridian and monitoring eye position with scleral search coils during psychophysical testing, and compared visual performance in lesioned and nonlesioned visual fields of each cat.

In an earlier study we examined the effects of area 18 lesions on spatiotemporal sensitivity and found drastically reduced sensitivity for detection of low spatial frequencies but intact spatial resolution (Pasternak and Maunsell, 1992). The lesions also eliminated the ability to discriminate the direction of motion at low spatial frequencies. This result suggested that area 18 was particularly important for the visibility of low spatial frequencies. The present study used simple gratings and more complex 
random dot patterns to examine the role of area 17 in visual function.

\section{Materials and Methods}

\section{Subjects}

Two adult female cats were used. During the period of behavioral testing they were maintained at $75-80 \%$ of their normal body weight. Water was continuously available in the home cage, and they received a daily supplement of Purina Chow.

\section{Stimuli}

Gratings. The stimuli were identical to those used in previous studies (Pasternak and Horn, 1991; Pasternak and Maunsell, 1992). Drifting, counterphase, or stationary gratings were displayed on a $1332 \mathrm{~A} \mathrm{HP}$ monitor (P-31 phosphor) at a distance of $42 \mathrm{~cm}$. The display was visible through a $6^{\circ}$ diameter circular aperture in a large $\left(60^{\circ}\right)$ white surround. The mean luminance of the display and its surround was set to $40 \mathrm{~cd} /$ $\mathrm{m}^{2}$. Stimulus duration followed a $350 \mathrm{msec}$ raised cosine temporal envelope.

Dynamic random dots. Dynamic random dot targets, used in the present study, were identical to those used recently by Pasternak et al. (1990). The stimuli, first introduced by Williams and Sekuler (1984), consisted of dots repeatedly displaced with a direction of motion chosen randomly from a uniform distribution of directions. The speed with which the dots were displaced was determined by the step size $(\Delta x)$ and the temporal interval between each step $(\Delta t)$. When the range of the distribution of directions was $360^{\circ}$ there was only local random motion of individual dots, and no coherent motion was seen. However, when the distribution was more limited, the dots appeared to flow coherently in the direction of the mean of the distribution. The salience of motion was manipulated by varying the range of the direction distribution (direction range), the proportion of the dots moving in random directions (\% motion signal), and the step size $(\Delta x)$. The spectral composition of the dot stimulus and the dimensions along which the stimulus was manipulated are shown in Figure 12.

The dots were displayed on the Apple 13-inch Color High Resolution Monitor (P22 Phosphor). The stimulus consisted of a $6^{\circ}$ diameter circular area containing 59 dots. The dot density was 2.1 dots/degree ${ }^{2}$, and the mean luminance of the display was $0.1 \mathrm{~cd} / \mathrm{m}^{2}$. Each dot subtended $0.05^{\circ}$ diameter and its luminance was set $3 \log$ units above detection threshold for human observers. Under these conditions there was no indication of streaks produced by moving dots.

\section{Control of eye position}

The details of procedures have been described elsewhere (Pasternak and Horn, 1991; Pasternak and Maunsell, 1992). Briefly, eye position was monitored with magnetic search coils (Remmel, 1984). A scleral search coil and head restraint device were implanted after the cat had adapted to the apparatus and learned the basic behavioral task. During behavioral testing, each cat was placed in a magnetic field generated by a 50 $\mathrm{cm}$ field coil and its head held firmly by means of a permanently implanted small vertical rod. Eye position was calibrated prior to each daily psychophysical session by rewarding the cat for fixating a dim laser spot projected onto the display surround. When the fixation spot appeared, the cat was required to position its gaze within a window centered on the target (Sparks and Sides, 1974) and maintain fixation within this window (usually $\pm 1^{\circ}$ ) for $700-1000 \mathrm{msec}$ in order to obtain food. The fixation spot was randomly moved to one of three positions along the horizontal meridian $\left(-10^{\circ}, 0^{\circ},+10^{\circ}\right)$, allowing the adjustment of signal gain and offset. Approximately 10-20 trials were usually needed for this calibration.

\section{Psychophysical procedure}

Each trial began with appearance of the fixation spot. When the cat fixated within $\pm 2^{\circ}$ of the spot and maintained fixation for $700 \mathrm{msec}$, a grating or a uniform field was presented simultaneously with a $450 \mathrm{mscc}$ tone. After termination of the tone, the cat was allowed to make a response. In the detection task, pressing the right pedal after the presentation of the vertical grating or the left pedal after presentation of the uniform field was rewarded with pureed beef. In the direction discrimination task, the right pedal response was rewarded if the grating (or dot pattern) moved to the right, and a response on the left pedal was rewarded if the stimulus moved to the left. In the orientation dis- crimination task, the cat was rewarded for pressing the right pedal if the grating was vertical and pressing the left pedal if the grating was horizontal (or of any other orientation). Incorrect responses were followed by a $6 \mathrm{sec}$ loud tone and no reward. A break in fixation or a response during stimulus (or uniform field) presentation resulted in termination of the trial. Responses during the $4 \mathrm{sec}$ intertrial interval delayed the beginning of the next trial. The sequence of grating/blank (or rightward/leftward motion; vertical/horizontal) presentation was randomized from trial to trial. To avoid position biases, a correction procedure was used: after three consecutive errors on one pedal, the same trial was repeated until the animal made a correct response (which was not included in the data analysis). Sessions consisted of 250 trials.

\section{Threshold measurements}

Thresholds were measured after the cat reached criterion performance (four consecutive sessions $>80 \%$ correct or three sessions $>90 \%$ correct) on tasks requiring discrimination between a high contrast moving grating and a uniform field (or high contrast gratings or dots of different orientations or moving in different directions). A staircase procedure was used to measure thresholds. In the detection task, three correct responses produced a decrease in contrast by $0.08 \log$ units (or increase in spatial frequency by 0.14 octaves), and each incorrect response made the stimulus more detectable by the same amount, either by an increase in contrast or a decrease in spatial frequency. During the presentation of the uniform field (blank) the correct response was rewarded and the incorrect response was followed by a loud tone, but the cat's performance had no effect on the staircase. The animal's performance during these blank trials averaged about 80-90\% (i.e., 10-20\% false alarms). A comparable procedure was employed in the direction and orientation discrimination tasks, with the exception that there were no blank trials, and responses on either of the pedals produced a change in staircase value. These procedures assured that the animal's performance was maintained at about $75 \%$ correct. Thresholds were taken as the stimulus value corresponding to $75 \%$ correct. At least three or four threshold determinations were made for each stimulus condition. Threshold measurements were performed at various eccentricities. When the target was viewed centrally the fixation spot was placed at the center of the display. For peripherally viewed targets, eccentricity was taken as the distance between the fixation spot and the center of the display. All stimuli were viewed binocularly.

\section{Cortical lesions}

Lesions were made by injecting ibotenic acid into physiologically identified loci of area 17. The animal was initially sedated with ketamine $(15 \mathrm{mg} / \mathrm{kg})$, and the trachea and a leg vein were cannulated. It was then anesthetized with $3 \%$ isoflurane and placed in a stereotaxic apparatus. A craniotomy was made over visual cortex on the right side. The dura mater was left intact and was covered with agar in sterile solution. The cat was then paralyzed (pavulon $5 / \mathrm{mg} / \mathrm{kg} / \mathrm{hr}$ or gallamine $7.5 \mathrm{mg} / \mathrm{kg}$ / $\mathrm{hr}$ ), artificially respired at a rate that kept end-tidal $\mathrm{CO}_{2}$ near $32 \mathrm{~mm}$ $\mathrm{Hg}$, and maintained on isoflurane for the remainder of the session. Body temperature was held near $37^{\circ} \mathrm{C}$ with a thermostatically controlled water pad. EKG, $\mathrm{CO}_{2}$, and temperature were continually monitored throughout the session.

The eyes were treated with atropine and Neo-Synephrine, and fitted with contact lenses to focus them on a tangent screen $114 \mathrm{~cm}$ from the animal. Each lens had an artificial pupil with a diameter of $3 \mathrm{~mm}$. The location of the optic disk was plotted on the screen by use of a reversing
ophthalmoscope. Multiunit recordings were made using glass-insulated $\mathrm{Pt} / \mathrm{Ir}$ microelectrodes. Signals were amplified, filtered, and monitored with an oscilloscope and audio amplifier. Receptive fields were plotted on the tangent screen using a hand-held projector. After identification of the region of cortex to be ablated, infusion of the paralytic was discontinued and ibotenic acid $(10 \mu \mathrm{g} / \mu \mathrm{l})$ was injected using a $10 \mu \mathrm{l}$ Hamilton syringe. Ten to thirteen closely spaced $1 \mu \mathrm{l}$ injections were made in each animal. Behavioral testing resumed about 1 week after the lesions and continued for a period of 24-36 months.

\section{Histology}

At the conclusion of behavioral testing, each cat was killed with an overdose of barbiturate and perfused with a phosphate buffer rinse, followed by $4 \%$ paraformaldehyde fixative. A second rinse was used to removed excess fixative. The brain was removed, blocked, and sectioned at a thickness of $40 \mu \mathrm{m}$. Pairs of adjacent sections at intervals 
Receptive field centers and injection sites

Figure 1. Electrophysiological mapping and placement of ibotenic acid injections. Locations of the receptive field centers in the visual field are shown by the circles. Each vertical electrode penetration is indicated by a number and the receptive fields of neurons encountered consecutively during each penetration are indicated by letters in alphabetical order. From 10 to 13 injections of $1 \mu \mathrm{l}$ of ibotenic acid were made in each animal, centered on a representation in the lower visual quadrant (filled circles)

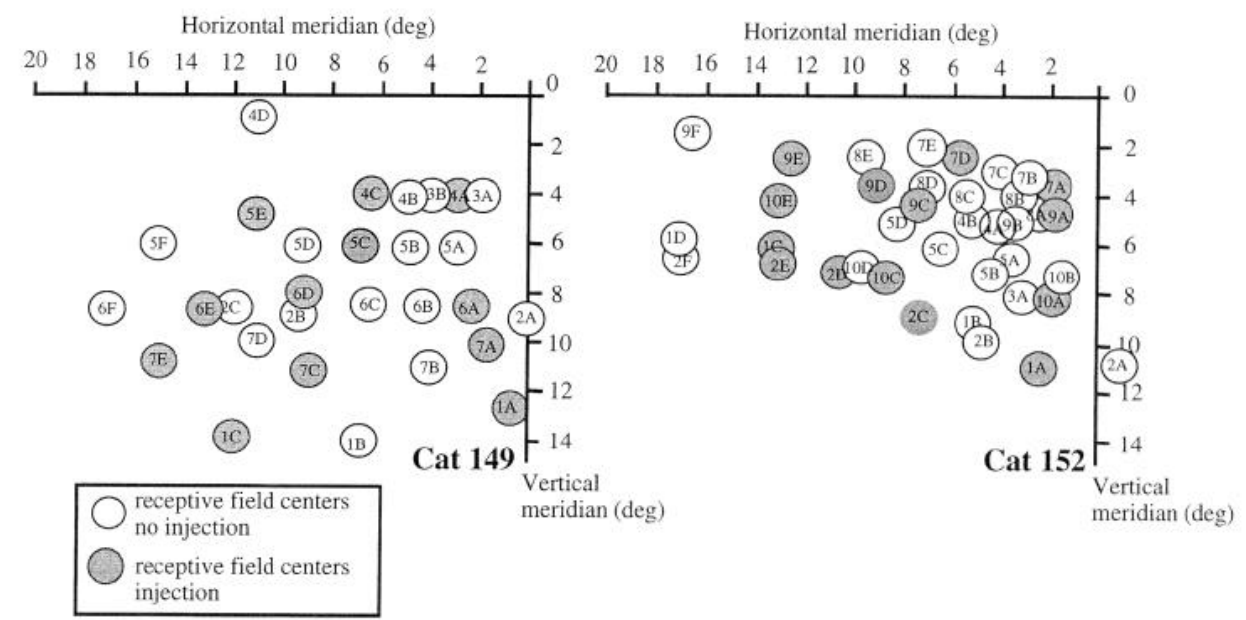

of $0.32 \mathrm{~mm}$ were stained for cytochrome oxidase (Wong-Riley, 1979) and with cresyl violet, respectively.

\section{Results}

\section{Extent of the lesions}

The results of the physiological mapping experiments performed at the time of injections are shown in Figure 1. The centers of all charted receptive fields are indicated by circles; the electrode penetrations are numbered in order and the receptive fields of cells encountered successively during a given penetration are labeled by letters in alphabetical order. At the end of the recording session, the injections were placed in the representation of the lower left quadrant of the visual field. Receptive fields at sites where ibotenic acid was injected are indicated by shading in Figure 1.

In cat 149 , the injections resulted in a confluent lesion extending approximately between P4.5 and A3 (in Horsley-Clark coordinates) along the medial surface of the splenial gyrus. The lesion was clearly visible as a pale zone in sections stained for cytochrome oxidase activity. Areas devoid of cytochrome oxidase activity exhibited complete neuronal cell loss and extensive gliosis in sections stained with cresyl violet. This is evident in a representative coronal section stained with cresyl violet (Fig. 2 ). In cat 152 , because the representation of the lower field was displaced 3-4 mm posteriorly, the lesion extended between P1 and P7. The location of the lower field representation in this cat is consistent with the pattern observed in a small proportion of cats by Tusa et al. (1978). In other respects (degree of cell loss; confluence of damage) this lesion was identical to that in cat 149.

Tracings of sections through the lesion for the two cats are shown in Figure 3.

The lesion, shown in black and by arrows, involved all cortical layers. The drawings indicate the borders between areas 17 and 18 which were established from the appearance of layers III and IV in sections stained for Nissl substance (Humphrey et al., 1985). The border with area 19 was determined from the appearance of layer IV in sections stained for cytochrome oxidase. In both animals, the lesion was confined exclusively to area 17 and did not appear to involve adjacent areas 18 and 19.

Complete reconstruction of the lesions in both cats are shown in Figure 4 as the medial view of the right hemisphere. The reconstructions show that the lesion was continuous.
We also examined the lateral geniculate nuclei of both cats stained with cresyl violet for the presence of cell loss. Areas of decreased cell density and apparent cell shrinkage within layers $A$ and A1 could be identified in sections stained with cresyl violet in both cats, at levels which, according to the published maps, contain a representation of the lower visual field (Sanderson, 1971). These areas had an unusually low density of cells and appeared to lack the largest neurons present in corresponding sections in the intact LGN. These abnormal areas are shown in line drawings of the right LGN in Figure 5.

\section{Spatiotemporal sensitivity: detection}

The locus and the extent of the lesion was mapped psychophysically in each cat by measuring contrast sensitivity for detecting a stationary $0.6 \mathrm{cycle} / \mathrm{degree}$ grating in various locations of the intact and lesioned quadrants of the visual field. The results of this mapping are shown in Figure 6 (top panel). Sensitivity measured at $8^{\circ}$ below the horizontal meridian (elevation $-8^{\circ}$ ) over a range of horizontal deviations from the vertical meridian (azimuths) is plotted in the two graphs on the left. In each animal, sensitivity dropped dramatically within the lesioned hemifield. In cat 152 sensitivity loss was maximal at $14^{\circ}$ left of the vertical meridian, while in cat 149 sensitivity was depressed at all tested locations left of the vertical meridian, up to $20^{\circ}$ azimuth.

To estimate the vertical extent of the lesion, similar measurements were performed at an azimuth of $14^{\circ}$ over a range of elevations. These data show (Fig. 6, top panel, right plots) that in both cats the maximal effect of the lesion was at an elevation of $-8^{\circ}$. In cat 149 , the lesion had no effect at an elevation of $-20^{\circ}$, suggesting a lower border of the lesion. Similarly, for cat 152 , the lesion effect was diminished, but not eliminated, at an elevation of $-14^{\circ}$, suggesting that the lower border of the lesion might be in the vicinity of about $-16^{\circ}$ to $-20^{\circ}$. Comparison of these results with the extent of the histologically reconstructed lesions demonstrates consistency between the psychophysically and anatomically determined loci of the lesions.

We used the results of these measurements to select the locus of the visual field for the more extensive measurements of the lesion effects described below. For both animals tests were centered on a locus at an elevation of $-8^{\circ}$ and an azimuth of $14^{\circ}$. 

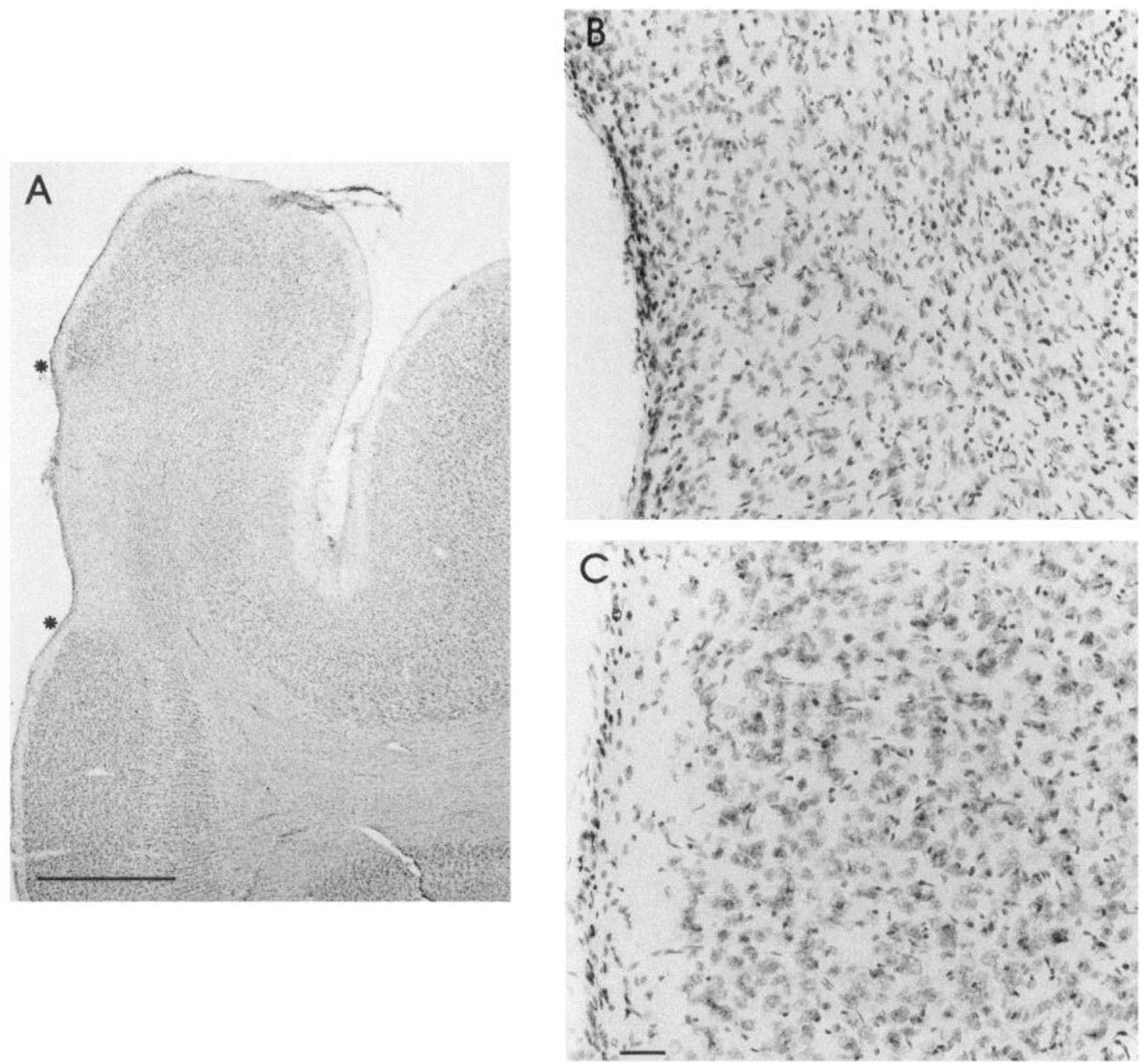

Figure 2. Histological coronal sections stained with cresyl violet showing the ibotenic acid lesion of area 17 in cat 149 . A, The lesion (demarcated by the two stars) is visible as a lightly staining region along the medial face of the lateral gyrus. $B$, Center of the lesioned area shown in $A$. $C$, Unlesioned area, slightly ventral to the lesion but still in area 17. Magnification the same as in $B$. Note the high density of neurons in $C$ and the lack of identifiable neurons in $B$. Scale bars: $A, 1 \mathrm{~mm} ; B$ and $C, 30 \mu \mathrm{m}$.

All of the data presented below were collected in this location, except as specifically noted.

Figure 7 shows contrast sensitivity functions measured at the above lesion locations (filled symbols) and at a control location in the corresponding portion of the opposite hemifield, for the detection of a stationary grating (left) and a grating drifting at $4.5 \mathrm{~Hz}$ (right). The points at the highest spatial frequency correspond to the cat's visual acuity, which was measured by varying spatial frequency at a single contrast of 0.63 .

The data show a modest loss (about $0.3 \log$ units) in sensitivity for the detection of stationary gratings at lower and intermediate spatial frequencies (Fig. 7, left plot). Surprisingly, there was no detectable loss in acuity. With the higher temporal frequency $(4.5 \mathrm{~Hz})$, both cats showed an even smaller loss in sensitivity at the lowest spatial frequencies $(0.1-0.2 \log$ units); thus, the deficit at lowest spatial frequencies was more pronounced at low temporal frequency, although temporal frequency had little or no effect on acuity.

Figure 8 shows how the deficits described above varied with temporal frequency of the drifting grating. The deficits measured at 1 and $4.5 \mathrm{~Hz}$ disappeared at a higher temporal frequency (18 $\mathrm{Hz}$ ). This result is consistent with the fact that neurons in area 17 respond poorly to low spatial frequencies modulated at high temporal frequencies (Movshon et al., 1978; Bisti et al., 1985; Ferster and Jagadeesh, 1991), and thus are unlikely to contribute to the detection of such patterns. Thus, the sensitivity loss at lower temporal frequencies suggests that area 17 contributes to the detection of low spatial frequencies at low but not at high temporal frequencies.

\section{Spatiotemporal sensitivity: orientation discrimination}

The preserved sensitivity to high spatial frequencies (see Fig. 7) appeared inconsistent with the preference of area 17 neurons for these frequencies. To rule out the use of undersampled (aliased) information we tested their high spatial frequency sensitivity with a discrimination between vertical and horizontal gratings. We used this task to measure visual acuity and to reassess the locus and the extent of the lesion previously determined with the detection task. The results of this mapping experiment are shown in the bottom plots of Figure 6. Visual acuity was mea- 
Cat 149

Figure 3. Cortical lesions. Line drawings of coronal sections of the brain of both cats showing the extent of the lesions. The sections are shown at about $1.5 \mathrm{~mm}$ intervals. Drawings were made from sections stained for cytochrome oxidase in which the lesion is seen as an area of reduced reactivity which is not appreciably darker than the white matter. The numbers indicate the approximate A-P coordinates. Lines perpendicular to the sections indicating the border between areas $17 / 18$ and 19 and the extent of area 17 were determined from the distinct appearance of layer 4 in sections stained for cytochrome oxidase and for the Nissl substance.

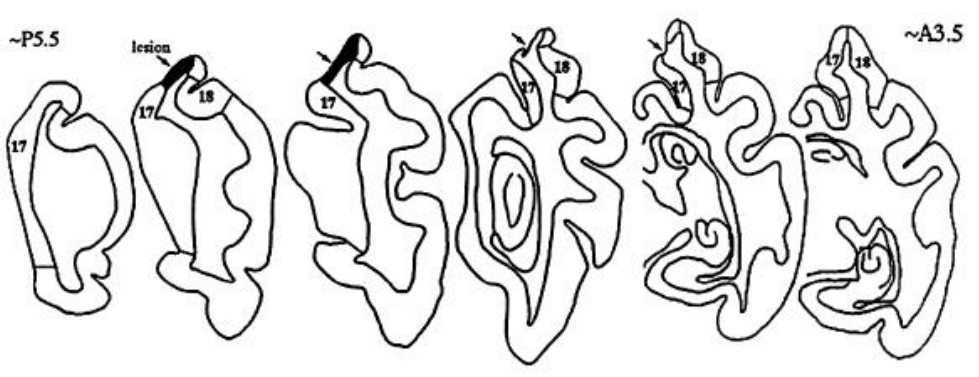

Cat 152

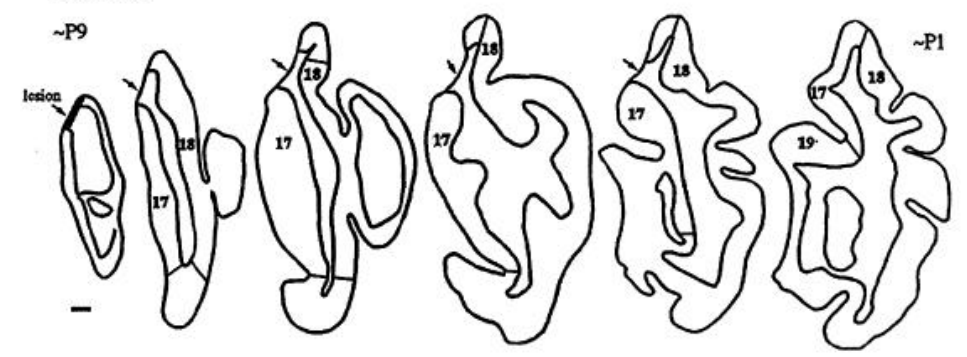

sured at several horizontal locations (azimuths) at an elevation of $-8^{\circ}$ (left graphs) and at three elevations along an azimuth of $14^{\circ}$ (right graphs) in the lesioned (filled symbols) and intact (open symbols) hemifield. The results were similar to those reported above for a detection task with gratings of intermediate spatial frequency. For both animals, the locus of maximal loss was at an elevation of $-8^{\circ}$ and an azimuth beyond $8-10^{\circ}$. Thus, the zone of reduced visual acuity as measured with an orientation discrimination task coincided with the zone of maximal sensitivity loss as determined with the detection task .

Contrast sensitivity for the lesioned and intact loci measured in the orientation discrimination task is shown in Figures 9 and 10. Contrast thresholds were measured with a stationary grating $(0 \mathrm{~Hz})$. Sensitivity loss was minimal at the lowest spatial frequency and increased at higher spatial frequency. The effect of temporal frequency was explored in one of the cats (cat 152). The data in Figure 10 show that the modest sensitivity loss observed at the lowest spatial frequency $(0.14$ cycle/degree) was
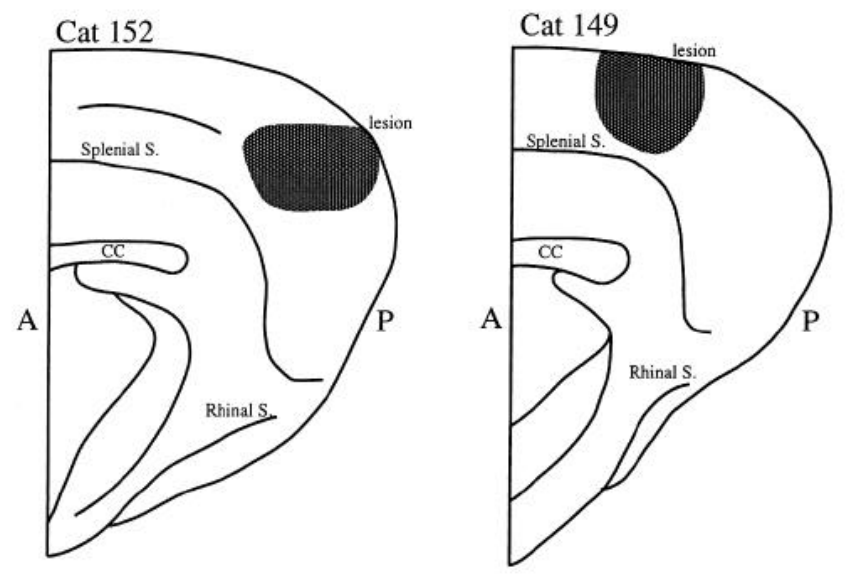

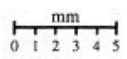

Figure 4. Full extent of each area 17 lesion as projected onto a medial view of the right hemisphere. significantly reduced at the highest temporal frequency. However, this normalization of performance at the highest temporal frequencies was not present at the higher spatial frequency $(0.3$ cycle/degree) (see Fig. 10, right plot).

Thus, striate cortex contributes little, if at all, to the orientation discrimination of low spatial frequency targets at high temporal
Cat 152
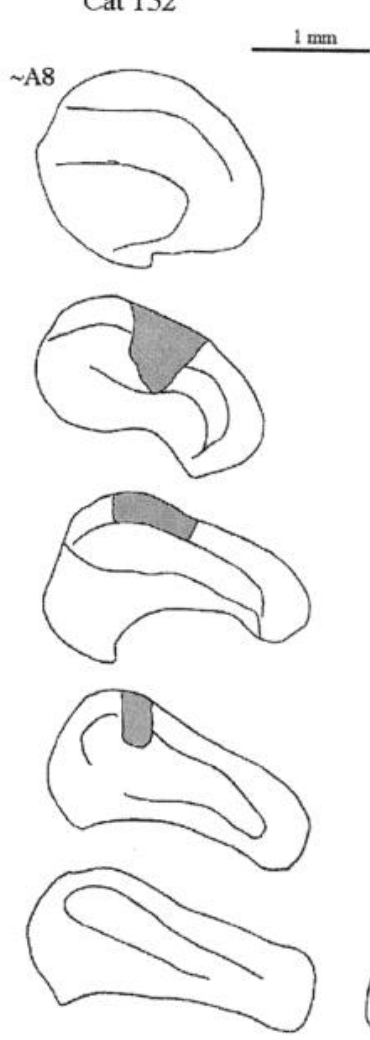

Cat 149
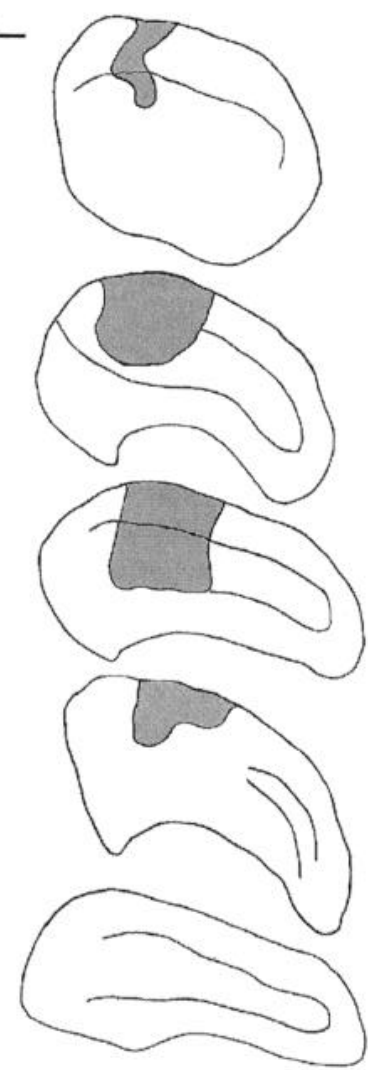

Figure 5. Line drawings of coronal sections showing the areas of cell loss and gliosis in the right LGN of both cats. 

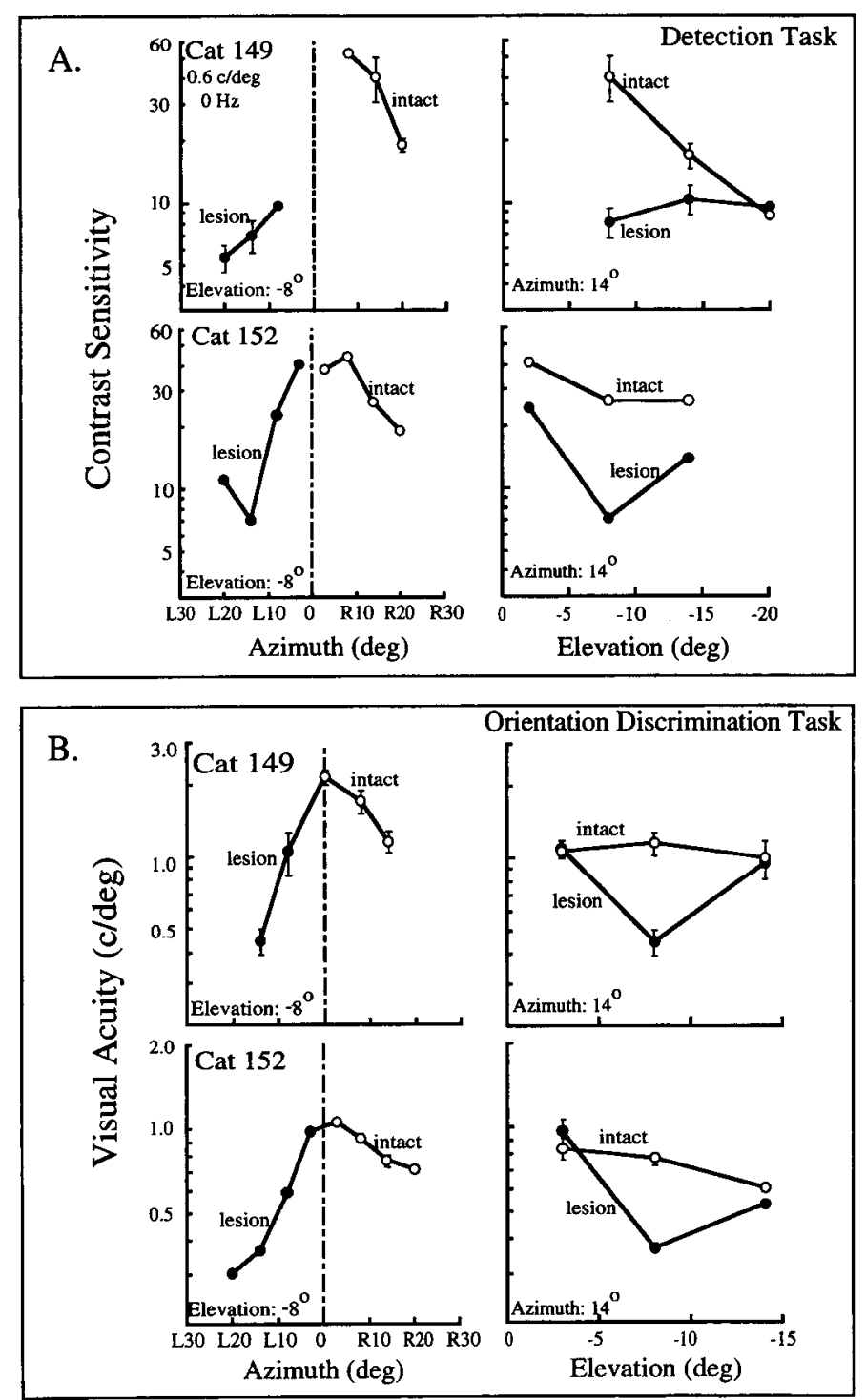

Figure 6. Psychophysical mapping of lesions. A, Detection task. Iefft plots, Contrast sensitivity measured at several locations along the azimuth in the right (intact) and the left (lesioned) hemifield. Elevation, $-8^{\circ}$. Right plots, Contrast sensitivity measured at several elevations below the horizontal meridian. Azimuth, $14^{\circ}$ into the right visual field (O) or left visual field (O). B, Orientation discrimination task. Left plots, Visual acuity measured at several locations along the azimuth. Right plots, Visual acuity measured at several elevations below the horizontal meridian. The cats discriminated between vertical and horizontal gratings. Error bars are SEM.

frequencies. Only at higher spatial frequencies does the contribution of area 17 become apparent.

\section{Orientation difference thresholds}

Possible effects of area 17 lesions on orientation sensitivity were assessed by measuring orientation difference thresholds. These measurements were performed with high contrast, low spatial frequency gratings, since at the lowest spatial frequency the sensitivity loss was very modest. The minimal discriminable difference in orientation was measured in a staircase procedure in which the cats discriminated between high contrast $(0.63)$, vertical and nonvertical, stationary, low spatial frequency $(0.14 \mathrm{cy}-$ cle/degree) gratings. We found that the lesions had no detectable

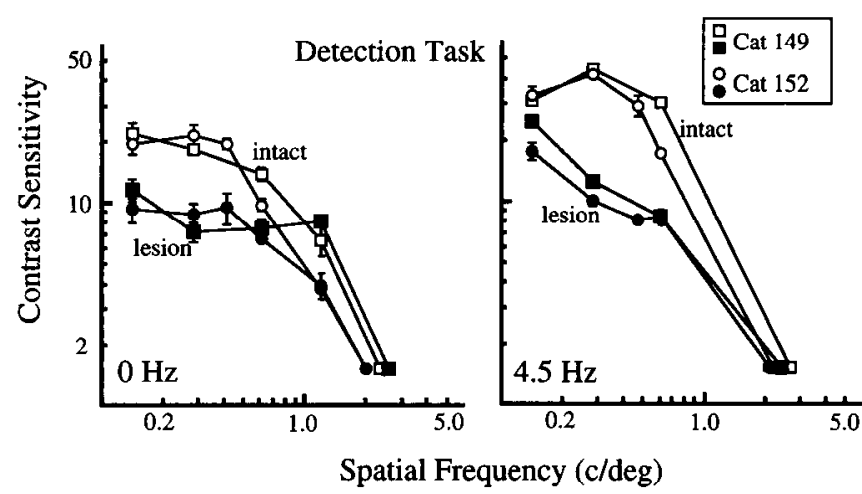

Figure 7. Detection task. Contrast sensitivity as a function of spatial frequency for detecting a stationary grating (left) or a grating drifting at $4.5 \mathrm{~Hz}$ (right). The data are shown for the intact $(0, \square)$ and the lesioned hemifield $(\boldsymbol{O}, \square)$. Target was centered at $14^{\circ}$ azimuth and $-8^{\circ}$ below the horizontal meridian. Error bars are \pm SEM.

effect on the accuracy of orientation discrimination. Specifically, the orientation difference threshold for cat 149 measured for the intact hemifield was $41.6^{\circ}$ (SEM \pm 1.75 ), while that measured for the lesioned hemifield was $37.4^{\circ}(\mathrm{SEM} \pm 4.02)$. The data were similar for cat 152 : intact hemifield, $51.7^{\circ}(\mathrm{SEM} \pm 3.7)$; lesioned hemifield, $58^{\circ}(\mathrm{SEM} \pm 2.3$ ). These measurements were performed at a $14^{\circ}$ azimuth and $-8^{\circ}$ elevation. It is important to note that when both cats were tested with identical gratings placed in the central visual field, orientation difference thresholds were substantially lower, ranging between 16 and $25^{\circ}$. The lack of detectable deficits measured in the portion of the visual field affected by area 17 lesion suggests that at low spatial frequencies, neurons in area 17 are not critical to the ability to discriminate small differences in orientation.

\section{Motion perception}

Possible contributions of area 17 to motion perception were studied most extensively in cat 152 , although a small number of tests were also performed in cat 149 and are shown when available.

Grating stimuli. The effect of area 17 lesions on the sensitivity of the directional mechanism was assessed in two tasks. In the first, sensitivity for detecting drifting low spatial frequency gratings was compared to that for detecting counterphase gratings. Normal animals and human observers typically have higher sen-

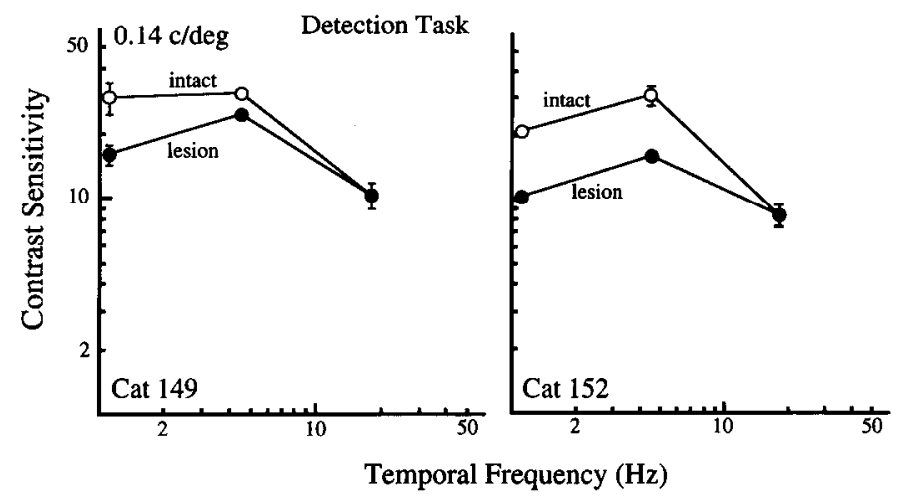

Figure 8. Detection task. Contrast sensitivity as a function of temporal frequency. Target location $14^{\circ}$ azimuth, $-8^{\circ}$ elevation. Error bars are SEM. 


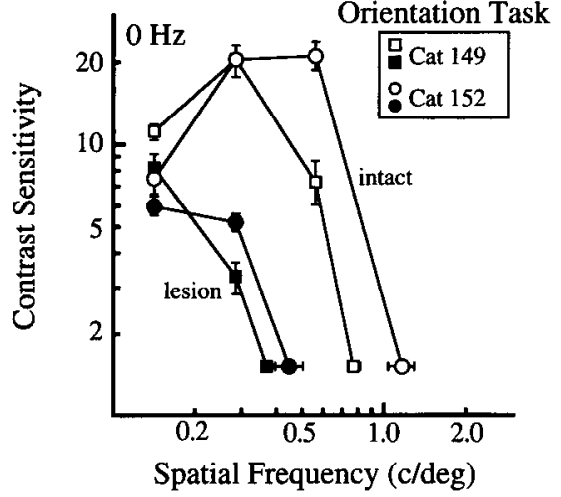

Figure 9. Orientation discrimination task. Contrast sensitivity for discriminating between vertical and horizontal stationary gratings measured in the intact $(O, \square)$ and lesioned $(\mathbf{Q}, \square)$ hemifield of the two cats. Target location $14^{\circ}$ azimuth, $-8^{\circ}$ elevation. Acuity measurements (the rightmost data points on each curve) were performed by varying spatial frequency at a contrast of 0.64 . Error bars are SEM.

sitivity for drifting gratings by less than a factor of two (Levinson and Sekuler, 1975; Watson et al., 1980; Pasternak, 1986). The results of such a comparison for cal 152 are shown in Figure $11 \mathrm{~A}$. In both the intact and lesioned hemifield the sensitivity for the drifting grating exceeded that for the counterphase grating, suggesting that at low spatial frequency, directional mechanisms in the lesioned hemifield are unaffected by the lesion. This conclusion is supported by results of the subsequent direction discrimination experiment, in which contrast sensitivity was measured over a range of temporal frequencies, as shown in Figure $11 B$.

At the lowest spatial frequency, cat 152 showed no detectable sensitivity loss over a broad range of temporal frequencies (speeds). At higher spatial frequencies, sensitivity loss was quite pronounced, but only at the lowest temporal frequency. Cat 149 also showed a slightly more pronounced deficit at the higher spatial frequency. These results demonstrate that the contribution of area 17 to direction discrimination is most pronounced for stimuli of higher spatial frequency moving at low speeds.

Dynamic random dot stimuli. Random dot stimuli were used to examine the contribution of area 17 to motion integration. Figure 12 illustrates the spectral composition of the dot stimuli and stimulus manipulations performed in this portion of the study.

Direction range. The ability to integrate local motion signals into a global motion percept was measured by varying the range of directions in the stimulus. The task was to discriminate between dot stimuli with global rightward versus leftward motion. Range thresholds for the intact and lesioned loci are shown in Figure 13 (top left). Surprisingly, the performance in the lesioned hemifield was superior to that in the intact hemifield; that is, the animal was able to integrate stimuli containing a range of directions twice as broad.

Motion signal. In a second task, the perception of global motion was measured with stimuli containing a variable proportion of coherently $\left(0^{\circ}\right.$ range $)$ and randomly moving dots $\left(360^{\circ}\right.$ range). The ability to extract motion of such stimuli was expressed as motion signal threshold. Again, the area 17 lesion resulted in superior performance; that is, the cat tolerated substantially higher levels of directional noise in the lesioned hemifield. Thus, by

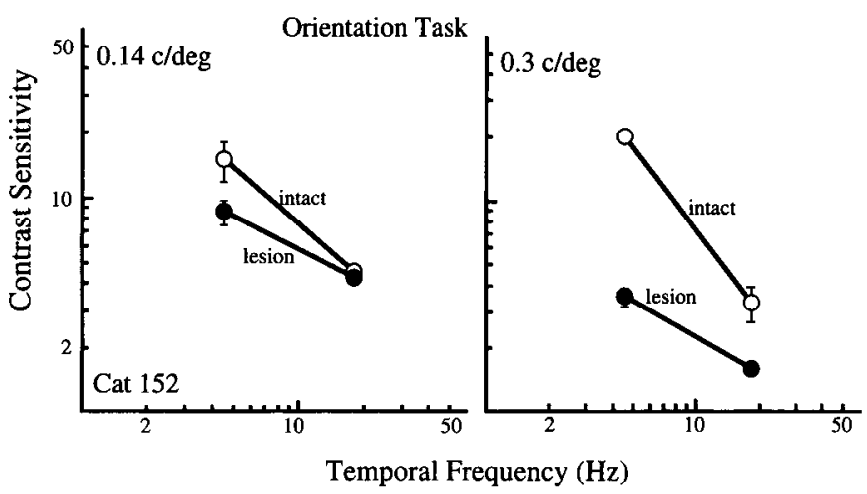

Figure 10. Orientation discrimination task. Contrast sensitivity at low and high temporal frequencies. Cat 152 . Target location $14^{\circ}$ azimuth, $-8^{\circ}$ elevation.

all three tests, the lesioned hemifield was superior at motion discrimination.

Maximal spatial displacement $\left(\mathrm{D}_{\max }\right)$. In this task, the spatial limit of the motion mechanism that underlies the perception of dot motion was measured. The dots were displaced coherently in a single direction and the maximal step size at which the animal was able to discriminate opposite directions was measured. The data in Figure 13 (bottom left) show that the $D_{\max }$ was about $0.3^{\circ}$ greater for the lesioned than for the intact hemifield.

Inferior motion thresholds in the intact hemifield could be due to masking by the higher spatial frequencies in the broadband dot stimulus (Cleary and Braddick, 1990). Although low spatial frequencies are dominant in the dot patterns used in the present study, many of the frequencies in the pattern are near the spatial resolution of the cats at the tested eccentricity (see Fig. 12D, arrows). Since the lesion produced a high spatial frequency loss (see Figs. 6, 9), masking by high spatial frequencies might affect only the intact hemifield, resulting in poorer motion thresholds. To test this hypothesis, the dot pattern was spatially filtered to remove higher spatial frequencies. The filtering was achieved by placing a diffuscr dircetly on the display screcn. The amplitude spectrum of the spatially filtered dot pattern is shown in Figure $12 D$. Filtering removed higher spatial frequency components. The right side of Figure 13 shows the performance on the three tasks measured with the filtered stimulus. Removal of higher frequencies resulted in improved performance for the intact hemifield, but produced little effect on performance in the lesioned hemifield. As a result, performance in the lesioned and the intact hemifields was nearly identical.

\section{Discussion}

Area 17 lesions resulted in a reduction in the ability to discriminate orientation and direction of motion of stimuli of higher spatial and lower temporal frequency. This deficit was revealed with tasks that required identification of grating orientation or the direction of its motion, but not by detection tasks. At the lower spatial frequencies, the ability to discriminate stimulus orientation and direction was largely preserved, particularly at higher temporal frequencies. Motion thresholds examined with dynamic random dot patterns not only survived area 17 lesion but were superior. This effect, however, was eliminated when the stimuli were filtered to remove higher spatial frequencies. 

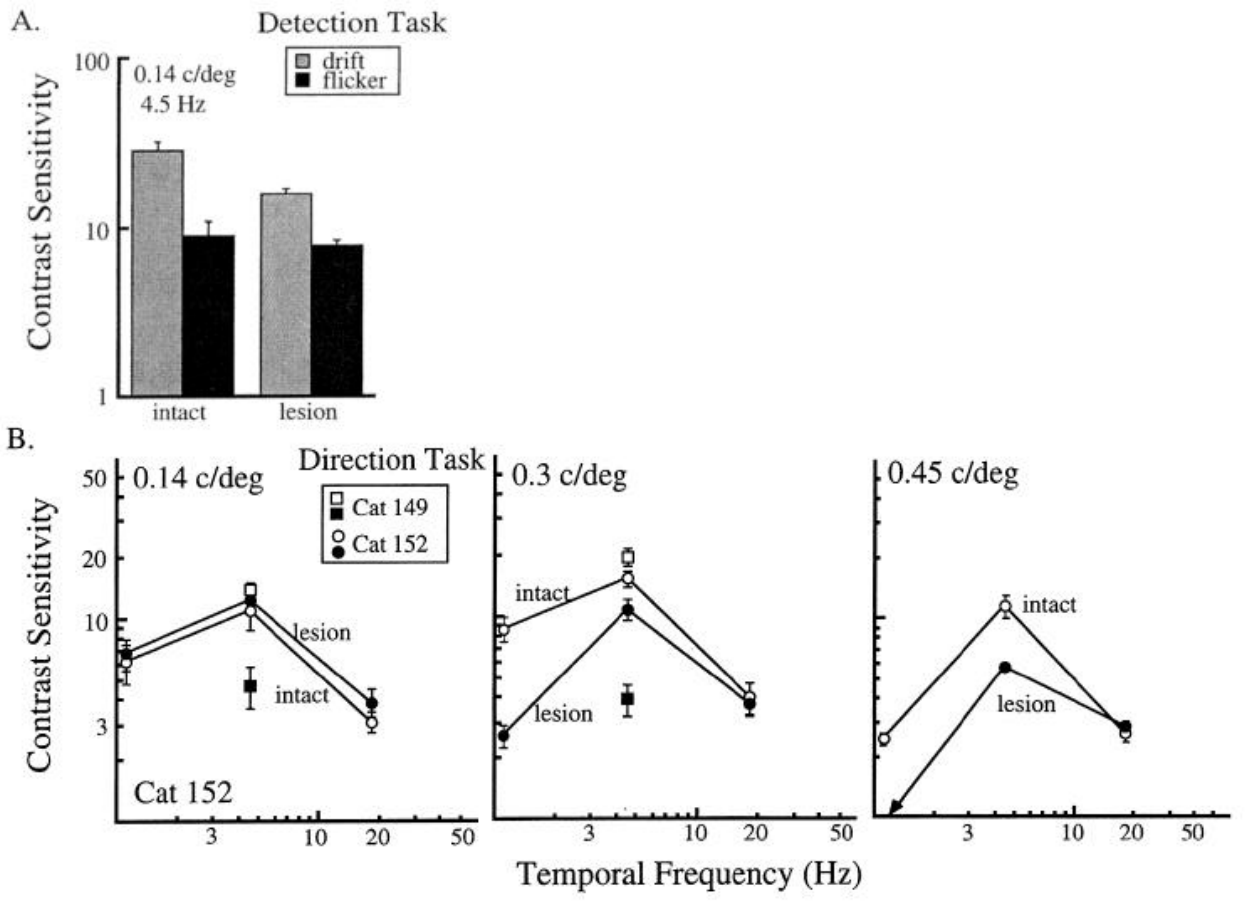

\begin{abstract}
Figure 11. A, Detection task. Contrast sensitivity for detecting drifting gratings (motion) and gratings modulated in counterphase (flicker) measured in cat 152. $B$, Direction discrimination task. Contrast sensitivity for discriminating between rightward and leftward motion. Target location $14^{\circ}$ azimuth, $-8^{\circ}$ elevation. Error bars are SEM.
\end{abstract}

\section{Methodological considerations}

The physical proximity of areas 17 and 18 imposed a number of limitations on our psychophysical experiments which should be kept in mind in evaluating the data. First, the lesions were placed in the peripheral representation of the visual field relatively far from the area centralis, the locus of highest sensitivity and resolution in the cat (Pasternak and Horn, 1991). Thus, all the psychophysical testing had to be performed at eccentricities where the sensitivity and resolution are inferior to that in cats tested with central fixation. The limited extent of the lesions (8$10^{\circ}$ ) also set a limit on the size of the target, since it had to be confined to the affected portion of the visual field. As a consequence, the number of cycles in gratings of low spatial frequency was relatively small, resulting in low contrast sensitivity (Savoy and McCann, 1975). These limitations were counterbalanced by our ability to use the intact hemifield as a control, which simplified the interpretation of lesion effects and problems associated with individual differences.

\section{The placement of lesions}

The physiological mapping used to guide the placement of the lesions enabled us to make lesions with well defined borders. Lesions were limited to an area of about $4 \times 5 \mathrm{~mm}$ (cat 149) and $4 \times 7 \mathrm{~mm}$ (cat 152) within area 17 on the medial face of the right hemisphere. In cat 149 the location of the lower visual field representation was consistent with standard published maps (Tusa et al., 1978). However, in cat 152 physiological mapping and subsequent anatomical reconstruction revealed that the map was shifted about $4 \mathrm{~mm}$ posterior. A similar shift has been noted in a small proportion of the cats studied by Tusa and his colleagues (Tusa et al., 1978). Despite this shift in cortical representation, the location of the area of cell loss in LGNs of both animals was fairly similar and involved the areas which, according to published maps (Sanderson, 1971), contain the representation of the lower left visual field.

\section{Spatiotemporal sensitivity in the absence of area 17}

Detection task. It was an unexpected finding that the simple detection of high spatial frequencies was not affected by the area 17 lesion. Neurons in area 17 prefer higher spatial frequencies and are the sole target of X-cells (Humphrey et al., 1985) which are presumed to carry high spatial frequency information to the cortex (Peichl and Wassle, 1979; Pasternak and Horn, 1991). On the other hand, neurons in the pathway originating in retinal Y-cells and projecting primarily to area 18 respond optimally to lower spatial frequencies. However, such neurons have frequency-doubled responses to counterphase gratings of spatial frequencies that are as high as those carried by linear X-cells (So and Shapley, 1981; Ferster and Jagadeesh, 1991). Similar nonlinear responses to high frequency gratings have also been observed in retinal W-cells (Rowe and Cox, 1993). The high frequency gratings detected in the lesioned portion of the visual field exceed the highest frequency of response expected from their sampling density (Nyquist frequency) for Y-cells (Wassle and Boycott, 1991), and probably W-cells. Thus, while they may signal the presence of gratings these cells are unlikely to code the periodicity and orientation of the gratings. The inability of cats with area 17 lesion to identify the orientation of high spatial frequency gratings supports the possibility that without area 17, they perceive the gratings nonveridically, using aliased signals provided by undersampling $\mathrm{Y}$ - and/or W-cells projecting to a number of cortical areas, including areas 18 and 19, and lateral suprasylvian cortex (Stone, 1983).

In both cats, the area 17 lesion produced an octave shift of the peak contrast sensitivity function toward lower spatial frequencies, and no loss at the highest temporal frequencies. This result is consistent with the loss of area 17 neurons which prefer higher spatial and lower temporal frequencies (Movshon et al., 1978). These properties of the residual contrast sensitivity function suggest that it could be determined in part by the activity of neurons in area 18 which remain visually responsive and largely retain their stimulus selectivity following lesions of area 

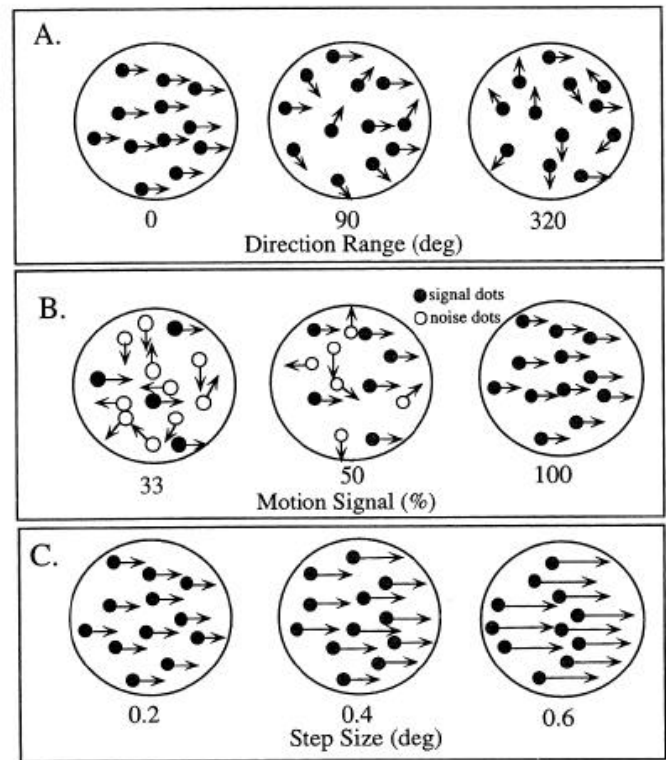

D.

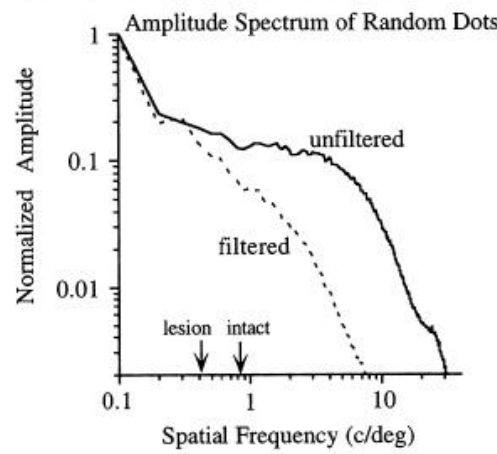

Figure 12. Parameters of random dot stimuli used to measure motion thresholds. All stimuli shown here appear to human observers to move rightward. $A$, Direction range. Maximal direction range at which opposite directions of motion could be reliably discriminated was measured. Direction range $=0^{\circ}$ : all dots move in a single direction. Direction range $=320^{\circ}$ : the direction of dot displacement is chosen from a distribution of directions of $320^{\circ}$. B , Motion signal. Stimuli consisted of signal dots moving in a single direction of motion and noise dots moving randomly. One hundred percent motion signal: all dots move in a single direction; $33 \%$ motion signal: $67 \%$ of dots are noise dots, while the others move in a predetermined single direction. $C$, Maximal spatial displacement $\left(D_{\max }\right)$. $D$, Amplitude spectrum of dot stimuli used in the experiment. Spatial resolution for the intact and lesioned loci of the visual field ( $\pm 14^{\circ}$ azimuth, $-8^{\circ}$ elevation) measured in the orientation task are indicated by arrows. Note that spatial filtering reduced the amplitudes of spatial frequencies above 0.2 cycle/degree.

17 (Dreher and Cottee, 1975; Sherk, 1978). This hypothesis is supported by a previous study in which we used a detection task to examine the effects of area 18 lesions (Pasternak and Maunsell, 1992). In that study, we found a severe loss of sensitivity at low spatial frequencies which was present at all temporal frequencies. This difference in the effects of area 17 and 18 lesions is consistent with the differences in physiological properties between the two areas (Movshon et al., 1978; Bisti et al., 1985; Ferster and Jagadeesh, 1991).

Thus, the detection task showed that area 17 is critical to the detection of low and intermediate spatial frequency gratings that are stationary or are temporally modulated at low rates. However, it failed to reveal any major contribution to the processing of high spatial frequencies.

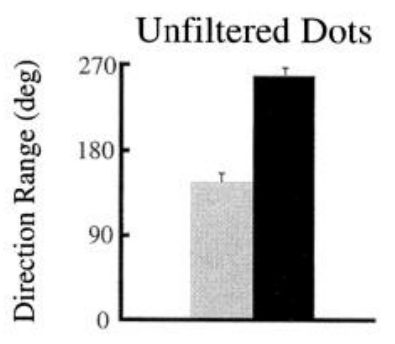

Filtered Dots
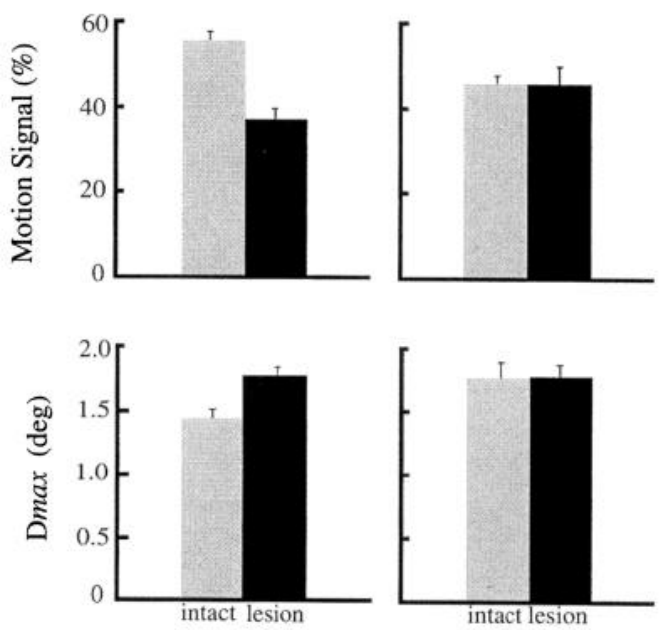

Figure 13. Motion thresholds measured with dynamic random dot stimuli (see Fig. 12). Range thresholds (top), motion signal thresholds (middle), and $D_{\max }$ (bottom) for discriminating opposite directions of motion with spatially unfiltered and filtered dot patterns. Target location $\pm 14^{\circ}$ azimuth, $-8^{\circ}$ elevation. Seventy-five dots in a $6^{\circ}$ target; dot density $2 /$ degree $^{2}$; speed, $60 \% \mathrm{sec} ; \Delta t, 40 \mathrm{msec}$; error bars, SEM.

Orientation discrimination task. This task has been used to eliminate the use of aliased information. As with the detection task, a small deficit found for low spatial frequency stationary gratings was less pronounced at high temporal frequencies (Figs. 8,9 ). Thus, the contribution of area 17 to the processing of low spatial frequencies appears to be minimal and limited primarily to stimuli of low temporal frequencies. This conclusion is further supported by the fact that orientation difference threshold for high contrast coarse gratings was unaffected by the lesion. This preservation of function is not surprising given that the unaffected frequencies are largely subserved by neurons located primarily in areas outside of area 17 , most likely in area 18 (Movshon et al., 1978). Although the ability to discriminate stimulus orientation was not examined in our previous study of the effects of area 18 lesion (Pasternak and Maunsell, 1992), the severe loss in detection sensitivity at low spatial frequencies suggests that the accuracy of orientation discrimination would also be severely compromised.

The inability to discriminate orientation at higher spatial frequencies after area 17 lesion is consistent with response properties of neurons in this area (Movshon et al., 1978), and demonstrates that area 17 neurons are critically important for the ability of cats to use high spatial frequency information in orientation discrimination tasks.

Direction discrimination task. Previous studies have shown that contrast sensitivity for direction discrimination is a reliable indicator of the activity of directionally selective cortical neurons (Pasternak et al., 1985). We used this measure to assess the role of directionally selective neurons in area 17 in direction 
discrimination. We explored contrast thresholds for direction over a broad range of spatiotemporal conditions in only one cat, but the more limited data for the other animal show a similar pattern of results. At low spatial frequencies, we found little or no loss in sensitivity for direction, particularly at high stimulus speeds, whereas at the higher spatial frequencies the deficit increased, but only for slow speeds. These results suggest that the contribution of area 17 to motion thresholds depends upon spatiotemporal stimulus parameters and is limited to stimuli of highcr spatial frequencies moving at low speeds.

We previously found that area 18 lesions result in a severe loss of sensitivity for detecting low spatial frequency gratings and an inability to discriminate the direction of motion of such gratings (Pasternak and Maunsell, 1992). This result complements the preservation of direction discrimination of coarse gratings and the loss of sensitivity for direction at higher spatial frequencies found here after area 17 lesion. The occurrence of complementary effects after lesions of areas 17 and 18 leads to the conclusion that directionally selective neurons in area 18 are likely to be critical for motion processing of coarse targets moving at high speeds while directionally selective neurons in area 17 may underlie the perception of motion of slowly moving finer gratings.

\section{Motion perception measured with random dot stimuli}

The limited contribution of area 17 neurons to motion perception of low spatial frequency stimuli was further confirmed with the dynamic random dots used to examine the integration of local directional signals. Such stimuli have previously been used to assess the role of various extrastriate cortical areas of cat and monkey in motion perception (Newsome and Paré, 1988; Rudolph and Pasternak, 1993; Pasternak and Merigan, 1994). These studies have shown that lesions of area MT in the monkey or of the lateral suprasylvian area in the cat produce only limited deficits in the perception of direction, suggesting that motion integration may be partially accomplished at earlier stages of cortical processing. The presence of intact motion integration in the absence of area 17 rules out the possibility that in the cat neurons in striate cortex are necessary for such integration to take place. However, it does not rule out the contribution of neurons in another "early" cortical area, area 18, to this function. This is particularly likely since the properties of area 18 neurons better match the spatiotemporal properties of the dot stimulus used in this study (see Fig. 12). Future studies examining the effects of selective lesions of area 18 on the perception of global motion will help resolve this question.

On the other hand, the superior performance on the three motion tasks in the lesioned portion of the visual field demonstrates some negative contribution of area 17 neurons to processing of random dot patterns. This contribution probably reflects only the high spatial frequency response of area 17 neurons rather than the analysis of motion direction per se (Morgan, 1992). The increase in maximal spatial displacement $\left(D_{\max }\right)$ after area 17 lesion, however, points to the possibility that the filter properties of area 17 neurons are likely to determine the spatial scale of the directional mechanism (Baker and Braddick, 1985; Mikami et al., 1986); and in the absence of these neurons, the spatial scale increased, reflecting the contribution of extrastriate neurons, such as those in area 18 with larger receptive fields (Movshon et al., 1978; Galli et al., 1988).

\section{Conclusion}

The present study provided strong evidence for a role of area 17 in processing of high spatial frequency signals temporally modulated or moved at low rates. Moreover, it demonstrated that at low spatial frequencies the processing of stimulus orientation or direction does not require striate cortex. This result, in conjunction with our previous demonstration for a role of area 18 in detection of low spatial frequencies, argues against areas 17 and 18 being specialized for processing of form and motion, respectively. Rather, it appears that areas 17 and 18 are specialized for processing both motion and form stimuli with different but somewhat overlapping spatiotemporal properties

\section{References}

Baker CL, Braddick OJ (1985) Eccentricity-dependent scaling of the limits for short range motion perception. Vision Res 25:803-812.

Berkley MA, Sprague JM (1979) Striate cortex and visual acuity functions in the cat. J Comp Neurol 187:679-702.

Bisti S, Carmignoto G, Galli L, Maffei L (1985) Spatial-frequency characteristics of neurons of area 18 in the cat: dependence on the velocity of the visual stimulus. J Physiol (Lond) 359:259-268.

Burke W, Dreher B, Michalski A, Cleland BG, et al. (1992) Effects of selective pressure block of $\mathrm{Y}$-type optic nerve fibers on the receptivefield properties of neurons in the striate cortex of the cat. Vis Neurosci $9: 47$.

Cleary R, Braddick OJ (1990) Direction discrimination for band-pass filtered random dot kinematograms. Vision Res 30:303-316.

Dreher B, Michalski A, Cleland BG, Burke W (1992) Effects of selective pressure block of $Y$-type optic nerve fibers on the receptivefield properties of neurons in area 18 of the visual cortex of the cat. Vis Neurosci 9:65.

Ferster D (1988a) X-and Y-mediated current sources in areas 17 and 18 of cat visual cortex. Vis Neurosci 4:135.

Ferster D, Jagadeesh B (1991) Nonlinearity of spatial summation in simple cells of areas 17 and 18 of cat visual cortex. J Neurophysiol 66:1667-1679.

Galli L, Chalupa L, Maffei L, Bisti S (1988) The organization of receptive fields in area 18 neurons of the cat varies with the spatiotemporal characteristics of the visual stimulus. Exp Brain Res 71: $1-7$.

Humphrey AL, Sur M, Uhlrich DJ, Sherman SM (1985) Terminal patterns of individual $\mathrm{X}$ - and $\mathrm{Y}$-cell axons in the visual cortex of the cat: projections to area 18 , to the $17 / 18$ border region, and to both areas 17 and 18. J Comp Neurol 233:190-212.

Lehmkuhle S, Kratz K, Sherman SM (1982) Spatial and temporal sensitivity of normal and amblyopic cats. J Neurophysiol 48:372-387.

Levinson E, Sekuler R (1975) The independence of channels in human vision selective for direction of movement. J Physiol (Lond) 250: 347-366.

Mikami A, Newsome WT, Wurtz, RH (1986) Motion selectivity in macaque visual cortex: spatio-temporal range of directional interactions in MT and V1. J Neurophysiol 55:1328-1339.

Morgan MJ (1992) Spatial filtering precedes motion detection. Nature 355:344-346.

Movshon JA, Thompson ID, Tolhurst DJ (1978) Spatial and temporal contrast sensitivity of neurons in areas 17 and 18 of the cat's visual cortex. J Physiol (Lond) 283:101-120.

Newsome WT, Paré EB (1988) A selective impairment of motion perception following lesions of the middle temporal visual area (MT). J Neurosci 8:2201-2211.

Orban GA, Kennedy H, Maes H (1981a) Response of movement of neurons in areas 17 and 18 of the cat: direction selectivity. J Neurophysiol 45:1059-1073.

Pasternak T (1986) The role of cortical directional selectivity in detection of motion and flicker. Vision Res 26:1187-1194.

Pasternak T, Horn K (1991) Spatial vision of the cat: variation with eccentricity. Vis Neurosci 6:151-158.

Pasternak T, Maunsell JHR (1992) Spatiotemporal sensitivity following lesions of area 18 in the cat. J Neurosci 12:4521-4529.

Pasternak T, Merigan WH (1994) Motion perception following lesions of the superior temporal sulcus in the monkey. Cereb Cortex 4:247259 . 
Pasternak T, Schumer RA, Gizzi MS, Movshon JA (1985) Abolition of cortical directional selectivity impairs visual behavior in cats. Exp Brain Res 61:214-217.

Pasternak T, Albano JE, Harvitt D (1990) The role of directionally selective neurons in the perception of global motion. J Neurosci 10: 3079-3086.

Peichl L, Wassle H (1979) Size, scatter and coverage of ganglion cell receptive field centres in the cat retina. J Physiol (Lond) 291:117141.

Remmel RS (1984) An inexpensive eye movement monitor using the scleral search coil technique. IEEE Trans Biomed Eng 31:388-390.

Rowe MH, Cox JF (1993) Spatial receptive-field structure of cat retinal W cells. Vis Neurosci 10:765-779.

Rudolph KK, Pasternak T (1993) Motion perception is affected by lesions of lateral suprasylvian cortex in the cat. Invest Ophthalmol Vis Sci [Suppl] 34:1030.

Savoy RL, McCann JJ (1975) Visibility of low-spatial-frequency sinewave targets: dependence on the number of cycles. J Opt Soc Am 65:343-350.

So YT, Shapley R (1981) Spatial tuning of cells in and around latera geniculate nucleus of the cat: $\mathrm{X}$ and $\mathrm{Y}$ relay cells and perigeniculate interneurons. J Neurophysiol 45:107-120.

Sparks DL, Sides JP (1974) Brain stem unit activity related to hori- zontal eye movements occurring during visual tracking. Brain Res 77:320-325.

Sprague JM, Levy J, DiBerardino A, Berlucchi G (1977) Visual cortical areas mediating form discrimination in the cat. J Comp Neurol $177: 441-488$.

Stone J (1983) Parallel processing in the visual system. New York: Plenum.

Stone J, Dreher B (1973) Projection of X- and Y-cells of the cat's lateral geniculate nucleus to areas 17 and 18 of visual cortex. J Neuruphysiol 36:551-567.

Tusa RJ, Palmer LA, Rosenquist AC (1978) The retinotopic organization of area 17 (striate cortex) in the cat. J Comp Neurol 177:213236.

Wassle H, Boycott BB (1991) Functional architecture of the mammalian retina. Physiol Rev 71:447-480.

Watson AB, Thompson PG, Murphy BJ, Nachmias J (1980) Summation and discrimination of gratings moving in opposite directions. Vision Res 20:341-348.

Williams DW, Sekuler R (1984) Coherent global motion perception from stochastic global motions. Vision Res 24:55-62.

Wong-Riley M (1979) Changes in the visual system of monocularly sutured or enucleated cats demonstrable with cytochrome oxidase histochemistry. Brain Res 171:11-28. 